\title{
Impact of Mental Health Background and Nutrition Intake on Medium-Term Weight Loss in Japanese Patients Undergoing Laparoscopic Sleeve Gastrectomy
}

\author{
Atsuhito Saiki $^{a}$ Rieko Kanai $^{\mathrm{b}}$ Shoko Nakamura ${ }^{\mathrm{a}}$ Sho Tanaka ${ }^{\mathrm{a}}$ \\ Rena Oka ${ }^{a}$ Yasuhiro Watanabe ${ }^{a}$ Takashi Yamaguchi ${ }^{a}$ Masahiro Ohira ${ }^{a}$ \\ Takashi Oshiro $^{c}$ Karin Hayashid ${ }^{d}$ Ichiro Tatsuno ${ }^{a}$ \\ ${ }^{a}$ Center of Diabetes, Endocrine and Metabolism, Toho University Sakura Medical Center, \\ Sakura, Japan; ${ }^{b}$ Department of Medical Nutrition, Toho University Sakura Medical Center, \\ Sakura, Japan; ' Department of Surgery, Toho University Sakura Medical Center, \\ Sakura, Japan; dDepartment of Neuropsychiatry, Toho University Sakura Medical Center, \\ Sakura, Japan
}

\section{Keywords}

Sleeve gastrectomy - Percent total weight loss - Weight regain - Mental disorder - Skeletal muscle

\begin{abstract}
Objectives: Bariatric surgery is the most effective weight loss therapy, and recently laparoscopic sleeve gastrectomy (LSG) is gaining popularity worldwide. On the other hand, patients undergoing bariatric surgery have a high prevalence of mental disorders. A Japanese nationwide survey reported high prevalence of mental disorders in patients with low percent total weight loss (\%TWL) and also in those with high \%TWL. The aim of this study was to investigate the relationship of 1-year \%TWL with background mental health status, 3-year outcomes, and nutrition intake in Japanese patients after LSG. Methods: This study was a single-center retrospective database analysis. A total of 89 Japanese patients who underwent LSG and were followed for 3 years were enrolled (mean age 41.9 years, baseline body mass index 44.9, baseline glycosylated hemoglobin, $\mathrm{HbA}_{1 c}, 7.0 \%$ ). The patients were divided into 3 groups according to 1 -year \%TWL as follows: $\leq 19.9 \%$ (insufficient group), $20.0-34.9 \%$ (average group) and $\geq 35.0 \%$ (excessive group). Psychosocial and nutritional status as well as physical data were collected from all patients. Results: The prevalence of mental disorders was $51.7 \%$, and 1-year \%TWL was $28.1 \%$ in all patients. No significant differences were observed in the changes in body weight and $\mathrm{HbA}_{1 c}$ between patients with and those without mental disorders. The prevalence of mental disorders was particularly high in the insufficient and excessive groups. In
\end{abstract}


the insufficient group, mood disorders and mental retardation/developmental disorders were frequent, and snacking and eating out habits were often observed. In the excessive group, the frequencies of mood disorders and binge eating were high, and a decrease in skeletal muscle mass due to low protein intake was observed. Furthermore, weight regain was shown 12 months after LSG in both groups. In the average group, there were fewer problems in weight loss outcomes, mental health, nutrition intake and body composition. Conclusions: Psychosocial and nutritional problems were often found not only in patients with insufficient weight loss, but also in those with seemingly "excellent" weight reduction. To improve long-term weight loss outcome and future health, a multidisciplinary approach focusing on mental health and nutrition is essential for patients undergoing bariatric surgery.

(C) 2020 The Author(s)

Published by S. Karger AG, Basel

\section{Introduction}

Obesity is associated with an increase in risk of obesity-related comorbidities such as type 2 diabetes, dyslipidemia, hypertension and sleep apnea syndrome. Obesity increases mortality and is therefore a serious problem globally. However, conventional approaches such as lifestyle modification, dietary control, increasing physical activity and pharmacotherapy are usually insufficient to achieve satisfactory weight reduction $[1,2]$. The cause of obesity is multifactorial, including genetic, social, economic, educational, environmental and psychological factors. Especially, individuals with a diagnosis of mental disorders are more likely to have difficulties losing weight than others in the population, which is associated with poorer physical health [3] and reduced life expectancy [4].

Bariatric surgery has been demonstrated to be the most effective weight loss therapy available. Laparoscopic Roux-en-Y gastric bypass was the most widely used bariatric procedures, while laparoscopic sleeve gastrectomy (LSG) as a single-stage procedure has recently gained popularity worldwide [5]. LSG has become a popular treatment option also in Japan, because LSG is the only bariatric procedure covered by the national health insurance. A nationwide survey conducted by the retrospective study group Japanese Survey of Morbid and Treatment-Resistant Obesity (J-SMART) reported that percent total weight loss (\%TWL) was $29.9 \%$ and complete diabetes remission rate was $75.6 \%$ after 2 years of LSG [6]. The prevalence of mental disorders at the time of preoperative evaluation in the J-SMART study was $26.3 \%$, compared with the prevalence of $20.9-37.8 \%$ reported by previous studies [7-9]. One of the notable findings of the J-SMART study was a high prevalence of mental disorders in patients with $<15 \%$ total weight loss and in those with $\geq 45 \%$ [6]. In other words, the relationship between frequency of mental disorders and \%TWL was a U-shaped curve. Further investigation is needed to clarify the psychophysiological background and medium-term outcome in patients with insufficient weight loss and also in those with seemingly "excellent" weight loss in the short term after LSG.

The aim of this study was to compare the mental health background and 3-year outcomes of body weight, metabolic parameters, nutrition intake and behaviors after LSG in Japanese patients stratified by 1-year \% TWL.

\section{Materials and Methods}

This study was a single-center retrospective database analysis. In the present study, a total of 89 (42 males and 47 females with a mean age of 41.9 years) Japanese patients who underwent LSG at the Toho University Sakura Medical Center between January 2010 and 
December 2016 with a follow-up period of 3 years were enrolled. LSG is the only bariatric procedure which is covered by the national health insurance in Japan, and all patients in this study received medical care under this system. The criterion for surgical indication was body mass index (BMI) higher than 35 with type 2 diabetes, dyslipidemia, hypertension and/or sleep apnea syndrome.

The following preoperative data were collected from medical records: age, anthropometric measurements, visceral fat area (VFA), subcutaneous fat area (SFA), skeletal muscle mass (SMM), blood pressure, glycosylated hemoglobin $\left(\mathrm{HbA}_{1 \mathrm{c}}\right)$, fasting blood glucose, fasting serum C peptide, lipid markers, liver function, renal function and apnea-hypopnea index. VFA was determined using computed tomography; the scan was performed at the umbilical level with the subject resting in the supine position. SFA was calculated by subtracting VFA from total fat area. SMM was measured by bioelectrical impedance analysis using InBody 720 (InBody Japan Co. Ltd., Tokyo, Japan) [10].

Information on nutrition, physical activity, mental disorders, intelligence and personality was also collected. Calorie intake and dietary composition were assessed by standardized interviews conducted by trained dieticians using a computerized database, and analysis of semiquantitative food records of 3 consecutive days, for each 2-week period. The criteria of lifestyle-related behaviors defined by the Japanese Ministry of Health, Labour and Welfare were as follows: frequent snacking was defined as more than $400 \mathrm{kcal} /$ day at least 3 times a week, regular physical exercise was defined as exercise for $30 \mathrm{~min}$ at least twice a week, and drinking habit was defined as consumption of more than $40 \mathrm{~g}$ of pure alcohol a day in males and $20 \mathrm{~g}$ in females.

In all patients, mental disorders were diagnosed preoperatively by skilled psychiatrists according to the criteria of the Diagnostic and Statistical Manual of Mental Disorder, 5th Edition, or the International Statistical Classification of Diseases and Related Health Problems, 10th Revision. Contraindications to LSG were basically persistent alcohol abuse, drug dependence and uncontrolled severe psychiatric illness [11]. Mental disorders included mental retardation, developmental disorders, binge eating and/or others. The patients were divided into 3 groups (insufficient group, average group and excessive group) according to 1-year $\%$ TWL based on the prevalence of mental disorders, and the optimal cutoff value and its reason are described in the Results section. Beck Depression Inventory II (BDI-II), Wechsler Adult Intelligence Scale (WAIS) III and Rorschach test were performed as intelligence and personality tests within general practice and were administered and interpreted by trained and skilled psychologists. The Rorschach test was administered using 10 different cards with ambiguous, symmetrical perceptual stimuli, known as "ink blots," and the test provides information on personality including affects, cognition, self-perception and interpersonal relationship.

SMM, VFA and SFA were measured at first visit, before operation, and 1, 2 and 3 years after LSG. Anthropometric measurements, blood samples and nutritional data were collected additionally at 1, 3, 6 and 9 months after LSG. The information of lifestyle-related behaviors was collected 1 year after LSG. The outcome of weight loss after LSG was evaluated as 1-year $\%$ TWL.

The results are expressed as means \pm SD. SPSS 15.0 (SPSS Inc., Chicago, IL, USA) was used in all statistical analyses. All parametric data were analyzed using Student's $t$ test. All nonparametric data were analyzed using the Mann-Whitney U test. Fisher's exact test was used to identify any significant difference between proportions and categorical variables. Comparisons among groups stratified by 1-year \%TWL were conducted using one-way ANOVA and Tukey's post hoc test. A two-sided $p$ value of 0.05 was considered statistically significant.

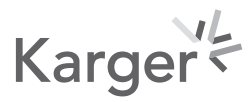


Table 1. Patient characteristics

\begin{tabular}{lcccc}
\hline & Total & Without \\
& & mental disorders & $\begin{array}{l}\text { With } \\
\text { mental disorders }\end{array}$ & $\begin{array}{l}p \\
\text { value }\end{array}$ \\
\hline Age, years & $41.9 \pm 9.0$ & $43.1 \pm 9.0$ & $40.7 \pm 8.9$ & $\mathrm{~ns}$ \\
Gender male/female & $42 / 47$ & $27 / 16$ & $15 / 31$ & 0.0083 \\
Height, cm & $164.2 \pm 10.0$ & $166.2 \pm 9.9$ & $162.3 \pm 9.7$ & $\mathrm{~ns}$ \\
Body weight, kg & $122.0 \pm 29.2$ & $127.3 \pm 33.7$ & $117.1 \pm 23.6$ & $\mathrm{~ns}$ \\
BMI & $44.9 \pm 8.2$ & $45.5 \pm 8.2$ & $44.4 \pm 8.2$ & $\mathrm{~ns}$ \\
Visceral fat area, cm ${ }^{2}$ & $218.7 \pm 96.1$ & $220.7 \pm 104.8$ & $216.8 \pm 66.6$ & $\mathrm{~ns}$ \\
Subcutaneous fat area, cm ${ }^{2}$ & $553.0 \pm 247.3$ & $565.5 \pm 270.5$ & $541.3 \pm 172.3$ & $\mathrm{~ns}$ \\
Systolic blood pressure, mm Hg & $134.5 \pm 21.0$ & $135.2 \pm 15.8$ & $133.9 \pm 15.3$ & $\mathrm{~ns}$ \\
Diastolic blood pressure, mm Hg & $80.6 \pm 13.3$ & $81.8 \pm 10.9$ & $79.4 \pm 9.6$ & $\mathrm{~ns}$ \\
FBG, mg/dL & $148.8 \pm 68.1$ & $141.2 \pm 55.7$ & $155.7 \pm 70.0$ & $\mathrm{~ns}$ \\
HbA 1 \% & $7.0 \pm 1.6$ & $6.8 \pm 1.6$ & $7.1 \pm 1.6$ & $\mathrm{~ns}$ \\
Serum CPR, ng/mL & $2.9 \pm 1.7$ & $2.7 \pm 1.1$ & $3.0 \pm 1.6$ & $\mathrm{~ns}$ \\
TC, mg/dL & $194.7 \pm 52.4$ & $187.2 \pm 33.9$ & $201.5 \pm 33.0$ & $\mathrm{~ns}$ \\
TG, mg/dL & $195.7 \pm 113.5$ & $183.9 \pm 106.5$ & $206.4 \pm 112.5$ & $\mathrm{~ns}$ \\
HDL-C, mg/dL & $46.6 \pm 14.6$ & $45.6 \pm 11.8$ & $47.5 \pm 14.0$ & $\mathrm{~ns}$ \\
AST, IU/L & $37.4 \pm 26.0$ & $38.1 \pm 27.4$ & $36.7 \pm 24.3$ & $\mathrm{~ns}$ \\
ALT, IU/L & $55.3 \pm 49.7$ & $53.3 \pm 45.4$ & $57.1 \pm 53.6$ & $\mathrm{~ns}$ \\
$\gamma$-GTP, IU/L & $57.0 \pm 45.0$ & $56.4 \pm 48.1$ & $57.6 \pm 41.6$ & $\mathrm{~ns}$ \\
Cr, mg/dL & $0.7 \pm 0.2$ & $0.7 \pm 0.1$ & $0.7 \pm 0.2$ & $\mathrm{~ns}$ \\
Uric acid, mg/dL & $6.2 \pm 1.6$ & $6.5 \pm 1.4$ & $5.9 \pm 1.1$ & $\mathrm{~ns}$ \\
Urine albumin, mg/g Cr & $173.6 \pm 555.3$ & $132.0 \pm 342.9$ & $212.4 \pm 878.5$ & $\mathrm{~ns}$ \\
Hemoglobin, g/dL & $14.4 \pm 3.0$ & $14.9 \pm 1.7$ & $14.0 \pm 1.4$ & $\mathrm{~ns}$ \\
AHI/h & $37.7 \pm 31.6$ & $41.4 \pm 26.1$ & $34.7 \pm 36.7$ & $\mathrm{~ns}$ \\
\hline
\end{tabular}

BMI, body mass index; $\mathrm{CPR}, \mathrm{C}$ peptide immunoreactivity; $\mathrm{HbA}_{1 \mathrm{c}}$, glycosylated hemoglobin; FBG, fasting blood glucose; TC, total cholesterol; TG, triglyceride; HDL-C, high-density lipoprotein cholesterol; AST, aspartate aminotransferase; ALT, alanine transaminase; $\gamma$-GTP, $\gamma$-glutamyltranspeptidase; $\mathrm{Cr}$, creatinine; AHI, apnea-hypopnea index; ns, not significant.

\section{Results}

Differences in Background Characteristics and 3-Year Outcome between Patients with and without Mental Disorders

At the first visit, the mean body weight, BMI and $\mathrm{HbA}_{1 \mathrm{c}}$ were $122.0 \mathrm{~kg}, 44.9$ and $7.0 \%$, respectively. Other background parameters are shown in Table 1. The mean prevalence of mental disorders was $51.7 \%$ (not significant), and there were no significant differences between patients with and those without mental disorders for all background parameters except for gender ratio.

The changes in body weight, $\mathrm{HbA}_{1 \mathrm{c}}$ and daily calorie intake at various time points are shown in Figure 1. After LSG, excessive weight loss was observed during the initial first year and the weight stabilized thereafter. At 1 year after LSG, mean body weight (BMI) declined to $87.0 \mathrm{~kg}$ (BMI) and mean \%TWL was $28.1 \%$ (Fig. $1 \mathrm{~A}$ ). Mean $\mathrm{HbA}_{1 \mathrm{c}}$ also decreased rapidly to $5.7 \%$ at 1 year and stabilized at $6.0 \%$ at 3 years (Fig. 1B). Mean daily calorie intake was 3,731.7 $\mathrm{kcal}$ at the first visit, decreased remarkably after LSG, and gradually increased during the postoperative period (Fig. 1C). There were no significant differences in the changes in body weight, $\mathrm{HbA}_{1 \mathrm{c}}$ and daily calorie intake between patients with and those without mental disorders.

\section{Difference in Mental Health Background among 1-Year \%TWL Groups}

Figure 2A shows the prevalence of mental disorders stratified by 1-year \%TWL. The relationship between frequencies of mental disorders and 1-year \%TWL was an almost U-shaped 


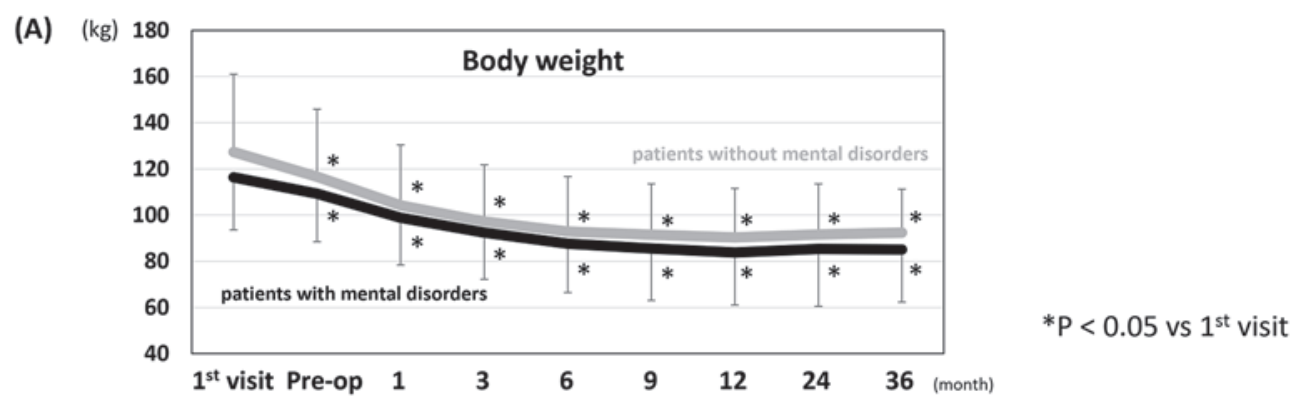

(B)

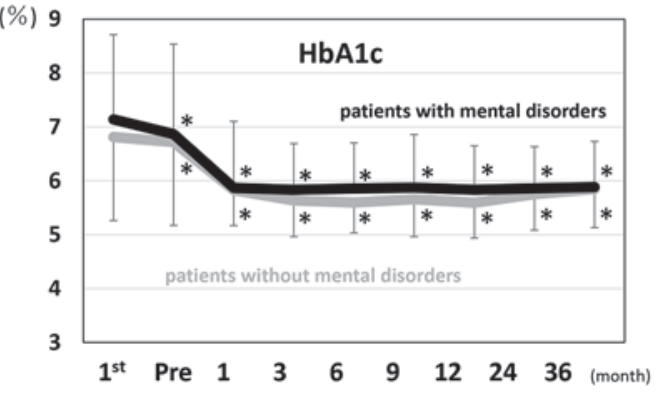

(C) (kcal/day) 6000

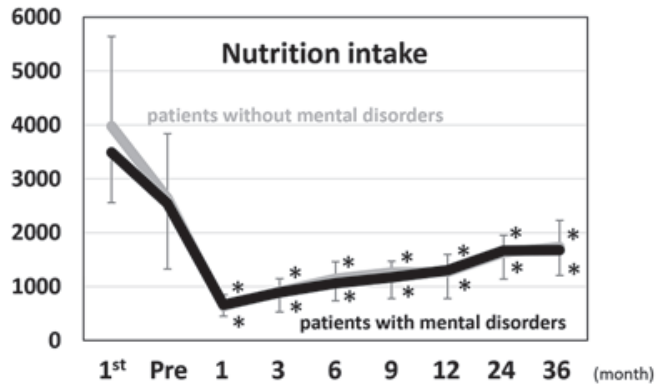

Fig. 1. Differences in 3-year outcome of body weight $(\mathbf{A}), \mathrm{HbA}_{1 \mathrm{c}}(\mathbf{B})$ and nutrition intake $(\mathbf{C})$ between patients with and those without mental disorders.

curve, and the trend was significant using Fisher's exact test $(p=0.0426)$. The optimal cutoff level of \%TWL was 19.9 and 35.0\%, and the reason is described in Figure $2 \mathrm{~B}$ and C. Therefore, the patients were divided into 3 groups according to 1 -year \%TWL as follows: $\leq 19.9 \%$ (insufficient group; $n=20$ ), 20.0-34.9\% (average group; $n=49$ ) and $\geq 35.0 \%$ (excessive group; $n=$ 20). There were no significant differences in physical parameters among these 3 groups (Table 2). In all subjects, mood disorders including depression and bipolar disorder were the principal diagnosis of mental disorder, constituting $29.5 \%$ of all mental disorders. In the insufficient, average and excessive groups, mood disorders were also the most frequent mental disorder, comprising around 30\% (Fig. 3). Mental disorders in the second and third places were mental retardation/developmental disorders and anxiety disorders, respectively, in the insufficient group, and binge eating and anxiety disorders in the excessive group. BDI-II is a widely used instrument for measuring the frequency and intensity of depressive symptoms. The BDI-II score was higher than normal limits in all 3 groups (Table 3 ). Regarding the WAIS-III, the mean verbal, performance and full-scale IQs in all subjects were within normal limits (90-109; Table 3). Lambda, which is an important variable in the Rorschach Comprehensive System, shows good validity in detecting tendency to simplification and defensiveness [12]. Lambda was higher only in the insufficient group (Table 3).

\section{Differences in 3-Year Outcomes of Body Weight, Body Composition, $H b A_{1 c}$ and Nutrition}

Intake among 1-Year \%TWL Groups

In the average group, weight regain was not observed, and \%TWL was 28.5 and $27.5 \%$ at 1 and 3 years, respectively, after LSG (Fig. 4A). On the other hand, 3-year \%TWL was significantly lower than 1-year \%TWL in the insufficient $(15.0$ vs. $7.3 \%, p<0.0001)$ and in the excessive group ( 40.3 vs. $34.7 \%, p=0.0128$ ). In all 3 groups, postoperative $\mathrm{HbA}_{1 \mathrm{c}}$ was signif- 


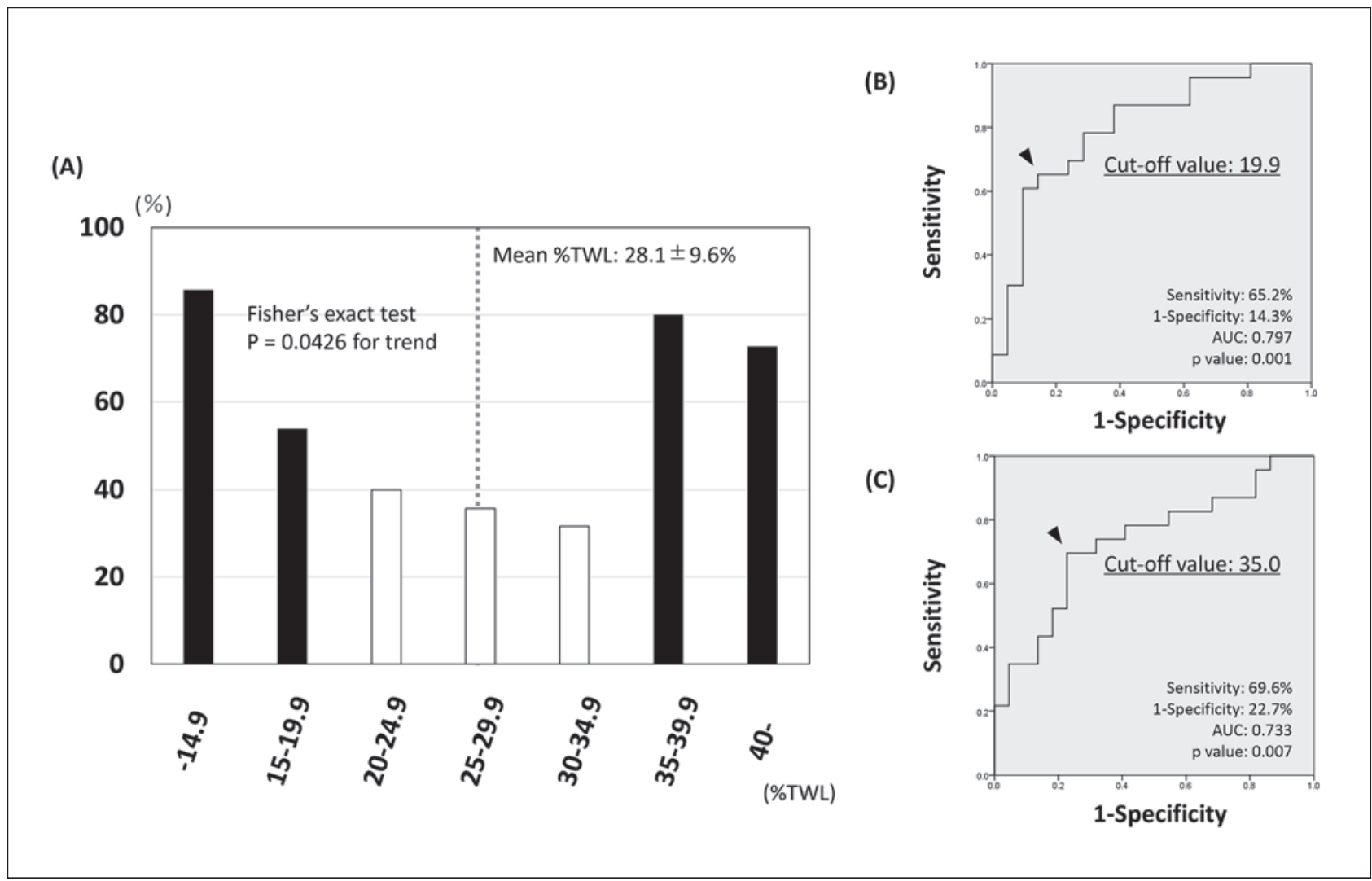

Fig. 2. A Prevalence of mental disorders stratified by 1-year \%TWL. Patients were divided into 2 groups based on mean \%TWL (28.1\%), and a receiver-operating characteristic (ROC) curve of \%TWL for the prevalence of mental disorders in each group was plotted. B, C ROC curve of \% TWL for the prevalence of mental disorders was generated for the below-average group (B) and for the above-average group (C).

icantly lower than that at the first visit (Fig. 4b). The complete remission rate of diabetes at 1 year after LSG was $52.6 \%$ in the insufficient group, $83.3 \%$ in the average group and $90.0 \%$ in the excessive group ( $p=0.0093$ by Fisher's exact test). VFA decreased significantly at 1 year after LSG in the 3 groups, and the decreases were no longer significant compared to baseline at 3 years in the insufficient and excessive groups (Fig. 4C). SMM also decreased at 1 year after LSG in the 3 groups and remained significantly lower at 3 years in the insufficient and excessive groups (Fig. 4D).

Changes in mean daily calorie, protein, fat and carbohydrate intakes over time after LSG in the 3 groups classified by 1-year \%TWL are shown in Figure 5. Mean daily calorie, fat and carbohydrate intakes in all postoperative periods were the highest in the insufficient group, although there were no significant differences (Fig. 5A, B, D). The AACE/TOS/ASMBS Guidelines recommend minimum protein intake of $60 \mathrm{~g}$ /day after bariatric surgery [13]. Daily protein intake in the insufficient and average groups recovered to $60 \mathrm{~g} /$ day at 9 months after LSG, whereas it took 24 months in the excessive group (Fig. 5C).

\section{Relationship between Postoperative Behavior and 1-Year \%TWL}

The relationship of postoperative eating and exercise behaviors with 1-year \%TWL is shown in Table 4. The percentages of frequent snacking and drinking habits were significantly higher in the insufficient group. There were no differences in the percentage of alcohol drinking habit and regular physical exercise among the 3 groups. 
Table 2. Physical parameters in the 3 groups

\begin{tabular}{|c|c|c|c|c|}
\hline & $\begin{array}{l}\text { Insufficient group } \\
(\% \mathrm{TWL}<19.9)\end{array}$ & $\begin{array}{l}\text { Average group } \\
\text { (\%TWL 20-34.9) }\end{array}$ & $\begin{array}{l}\text { Excessive group } \\
(\% \mathrm{TWL} \geq 35.0)\end{array}$ & $p$ value \\
\hline Age, years & $042.0 \pm 9.2$ & $042.6 \pm 9.3$ & $040.0 \pm 8.1$ & ns \\
\hline Gender male/female & $00.11 / 9$ & $00.25 / 24$ & $00.06 / 14$ & ns \\
\hline Height, cm & $164.1 \pm 12.7$ & $164.4 \pm 9.7$ & $163.6 \pm 7.9$ & ns \\
\hline Body weight, kg & $117.2 \pm 27.8$ & $121.8 \pm 29.5$ & $127.4 \pm 30.6$ & ns \\
\hline BMI & $043.2 \pm 7.6$ & $044.7 \pm 8.1$ & $047.3 \pm 8.7$ & ns \\
\hline Visceral fat area, $\mathrm{cm}^{2}$ & $205.3 \pm 62.4$ & $226.1 \pm 94.6$ & $214.4 \pm 89.1$ & ns \\
\hline Subcutaneous fat area, $\mathrm{cm}^{2}$ & $474.0 \pm 175.4$ & $560.7 \pm 249.6$ & $610.2 \pm 187.8$ & ns \\
\hline Systolic blood pressure, mm Hg & $131.5 \pm 12.8$ & $135.9 \pm 16.5$ & $134.5 \pm 15.7$ & ns \\
\hline Diastolic blood pressure, $\mathrm{mm} \mathrm{Hg}$ & $079.8 \pm 9.3$ & $081.1 \pm 11.1$ & $080.0 \pm 9.7$ & ns \\
\hline $\mathrm{FBG}, \mathrm{mg} / \mathrm{dL}$ & $141.1 \pm 54.9$ & $159.0 \pm 66.9$ & $131.2 \pm 60.9$ & ns \\
\hline $\mathrm{HbA}_{1 \mathrm{c}}, \%$ & $006.9 \pm 1.3$ & $007.2 \pm 1.8$ & $006.4 \pm 1.1$ & ns \\
\hline Serum CPR, ng/mL & $003.1 \pm 1.4$ & $002.9 \pm 1.5$ & $002.5 \pm 0.8$ & ns \\
\hline $\mathrm{TC}, \mathrm{mg} / \mathrm{dL}$ & $206.4 \pm 40.2$ & $188.5 \pm 32.6$ & $198.8 \pm 28.8$ & ns \\
\hline $\mathrm{TG}, \mathrm{mg} / \mathrm{dL}$ & $203.6 \pm 136.4$ & $197.9 \pm 102.6$ & $183.3 \pm 104.3$ & ns \\
\hline HDL-C, mg/dL & $043.8 \pm 9.0$ & $047.4 \pm 15.1$ & $047.0 \pm 10.3$ & ns \\
\hline AST, IU/L & $040.8 \pm 21.2$ & $037.3 \pm 26.0$ & $034.5 \pm 29.3$ & ns \\
\hline ALT, IU/L & $053.7 \pm 32.0$ & $055.4 \pm 45.0$ & $056.4 \pm 71.3$ & ns \\
\hline$\gamma$-GTP, IU/L & $062.2 \pm 42.6$ & $060.7 \pm 50.1$ & $043.6 \pm 28.0$ & ns \\
\hline $\mathrm{Cr}, \mathrm{mg} / \mathrm{dL}$ & $000.7 \pm 0.3$ & $000.7 \pm 0.1$ & $000.7 \pm 0.2$ & ns \\
\hline Uric acid, mg/dL & $458.6 \pm 1406.9$ & $135.6 \pm 326.8$ & $029.1 \pm 34.4$ & ns \\
\hline Urine albumin, mg/g Cr & $006.2 \pm 1.6$ & $006.2 \pm 1.3$ & $006.0 \pm 1.0$ & ns \\
\hline Hemoglobin, g/dL & $014.0 \pm 1.9$ & $014.7 \pm 1.4$ & $014.1 \pm 1.6$ & ns \\
\hline $\mathrm{AHI} / \mathrm{h}$ & $025.3 \pm 23.2$ & $046.8 \pm 33.7$ & $027.6 \pm 31.1$ & ns \\
\hline
\end{tabular}

BMI, body mass index; $\mathrm{HbA}_{1 \mathrm{c}}$, glycosylated hemoglobin; CPR, C peptide immunoreactivity; FBG, fasting blood glucose; TC, total cholesterol; TG, triglyceride; HDL-C, high-density lipoprotein cholesterol; AST, aspartate aminotransferase; ALT, alanine transaminase; $\gamma$-GTP, $\gamma$-glutamyltranspeptidase; $\mathrm{Cr}$, creatinine; AHI, apnea-hypopnea index; ns, not significant.
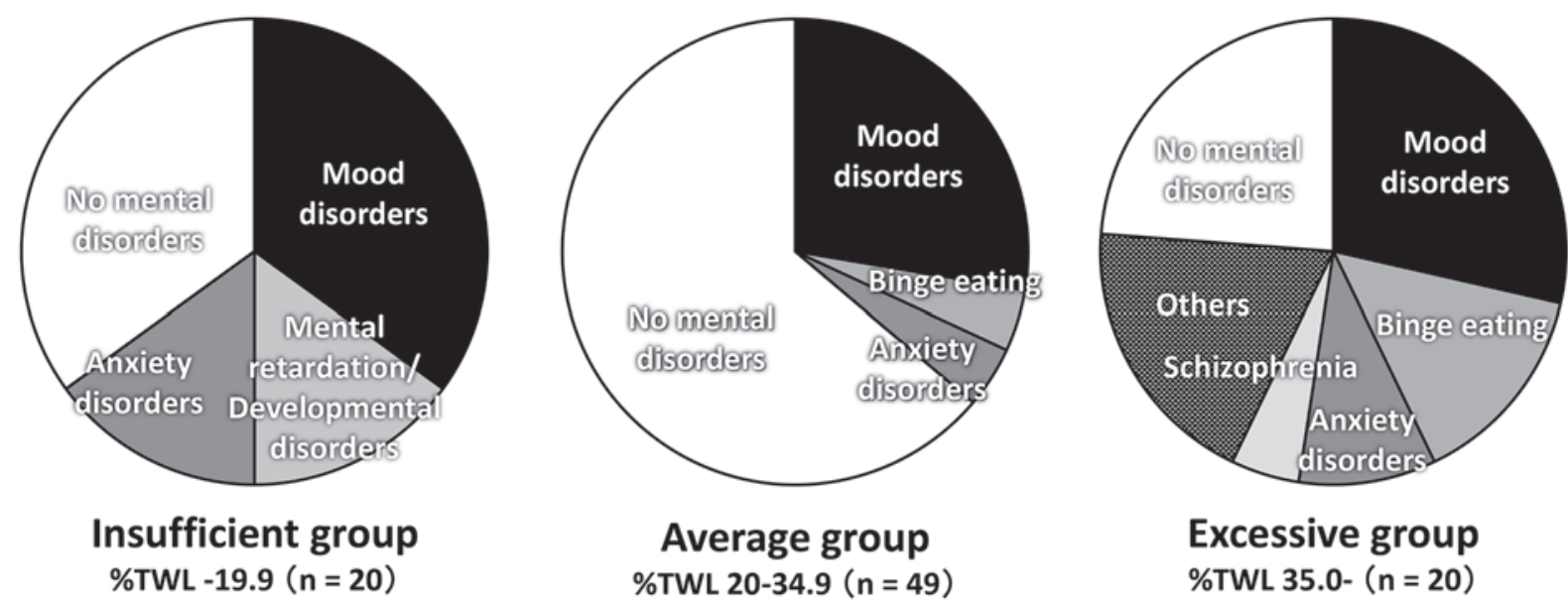

Fig. 3. Differences in principal diagnoses of mental disorders among 3 groups classified by 1-year \%TWL. 
Table 3. Mental disorders assessed in the 3 groups

\begin{tabular}{lllll}
\hline & $\begin{array}{l}\text { Insufficient group } \\
\text { (\%TWL <19.9) }\end{array}$ & $\begin{array}{l}\text { Average group } \\
\text { (\%TWL 20-34.9) }\end{array}$ & $\begin{array}{l}\text { Excessive group } \\
(\% \mathrm{TWL} \geq 35.0)\end{array}$ & $p$ value \\
\hline $\begin{array}{l}\text { Prevalence of any mental disorder, \% } \\
\text { BDI-II (normal upper limit: 10) }\end{array}$ & 65.0 & 36.7 & 75.0 & 0.0062 \\
$\begin{array}{l}\text { WAIS-III (normal range: 90-109) } \\
\quad \text { Full-Scale IQ }\end{array}$ & $17.0 \pm 11.9$ & $12.4 \pm 10.7$ & $15.8 \pm 10.4$ & $\mathrm{~ns}(0.0588)$ \\
$\quad \begin{array}{l}\text { Verbal IQ } \\
\quad \text { Performance IQ }\end{array}$ & $91.2 \pm 15.5$ & $98.7 \pm 16.3$ & $92.3 \pm 14.7$ & $\mathrm{~ns}$ \\
$\begin{array}{l}\text { Rorschach test } \\
\quad \text { Lambda (normal upper limit: 0.99) }\end{array}$ & $93.9 \pm 14.2$ & $98.8 \pm 14.9$ & $94.4 \pm 13.2$ & $\mathrm{~ns}(0.0956)$ \\
\hline
\end{tabular}

BDI-II, Beck Depression Inventory II; WAIS III, Wechsler Adult Intelligence Scale III; ns, not significant.

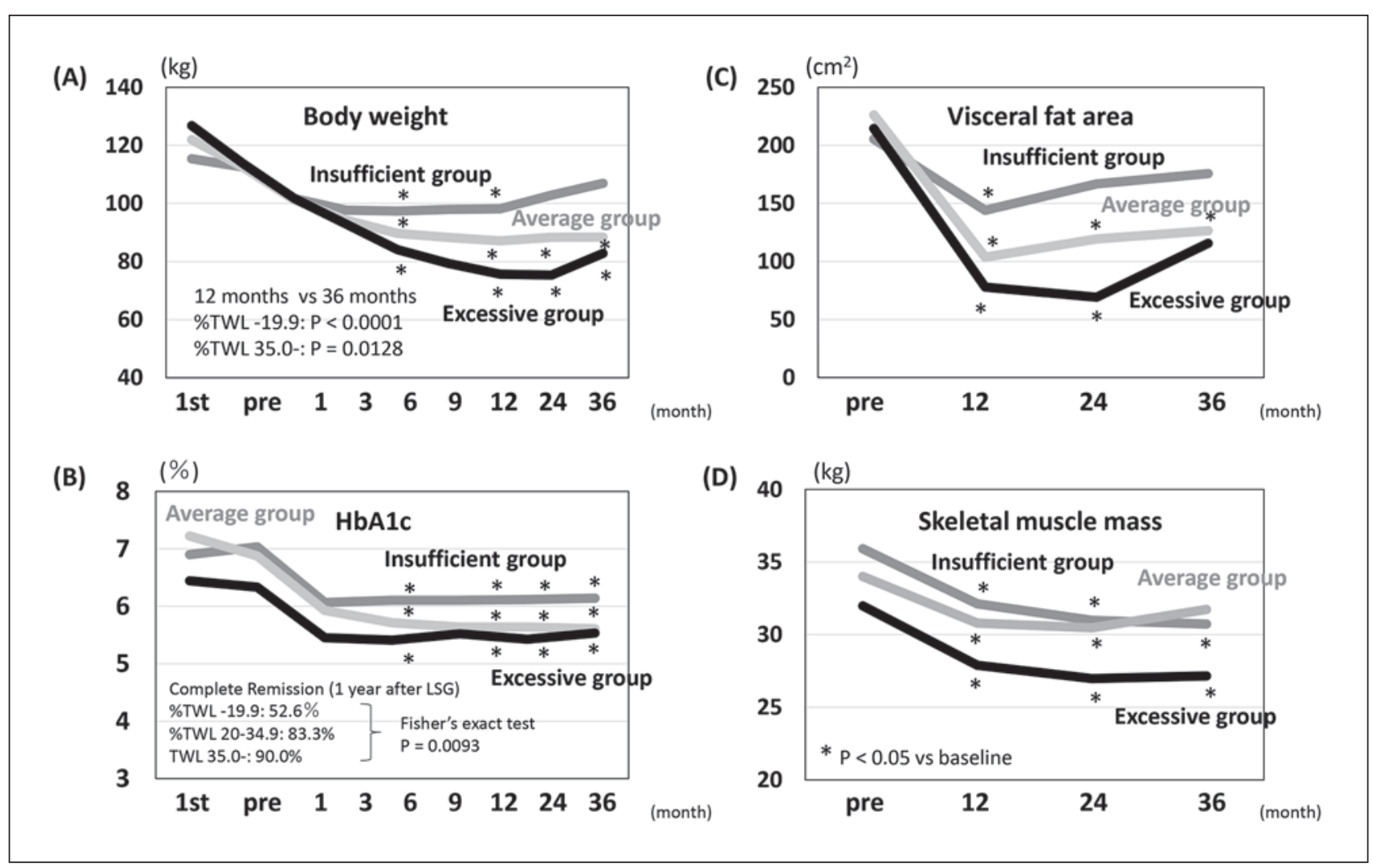

Fig. 4. Differences in 3-year outcomes of body weight, $\mathrm{HbA}_{1 \mathrm{c}}$ and body composition among groups classified by 1-year \%TWL. Changes in body weight $(\mathbf{A}), \mathrm{HbA}_{1 \mathrm{c}}(\mathbf{B})$, visceral fat area $(\mathbf{C})$ and skeletal muscle mass (D) are shown.

\section{Discussion}

Patients who undergo bariatric surgery are known to have a high prevalence of mental disorders. However, the evidence regarding the association between preoperative mental health conditions and postoperative weight loss remains controversial. In Japan, the multicenter J-SMART study was the first to investigate the relationship between the prevalence of 
Table 4. Postoperative eating and exercise behaviors in the 3 groups

\begin{tabular}{|c|c|c|c|c|}
\hline & $\begin{array}{l}\text { Insufficient group } \\
(\% \mathrm{TWL}<19.9)\end{array}$ & $\begin{array}{l}\text { Average group } \\
\text { (\%TWL 20-34.9) }\end{array}$ & $\begin{array}{l}\text { Excessive group } \\
(\% \mathrm{TWL} \geq 35.0)\end{array}$ & $p$ value \\
\hline Frequent snacking, \% & 70.0 & 14.3 & 15.0 & $<0.0001$ \\
\hline Drinking habit, $\%$ & 60.0 & 34.7 & 15.0 & 0.0119 \\
\hline Eating out at least 3 times a week, \% & 30.0 & 12.2 & 5.0 & ns $(0.0640)$ \\
\hline Alcohol drinking habits, $\%$ & 5.0 & 10.2 & 10.0 & ns \\
\hline Regular physical exercise, \% & 10.0 & 16.3 & 25.0 & ns \\
\hline
\end{tabular}

ns, not significant.

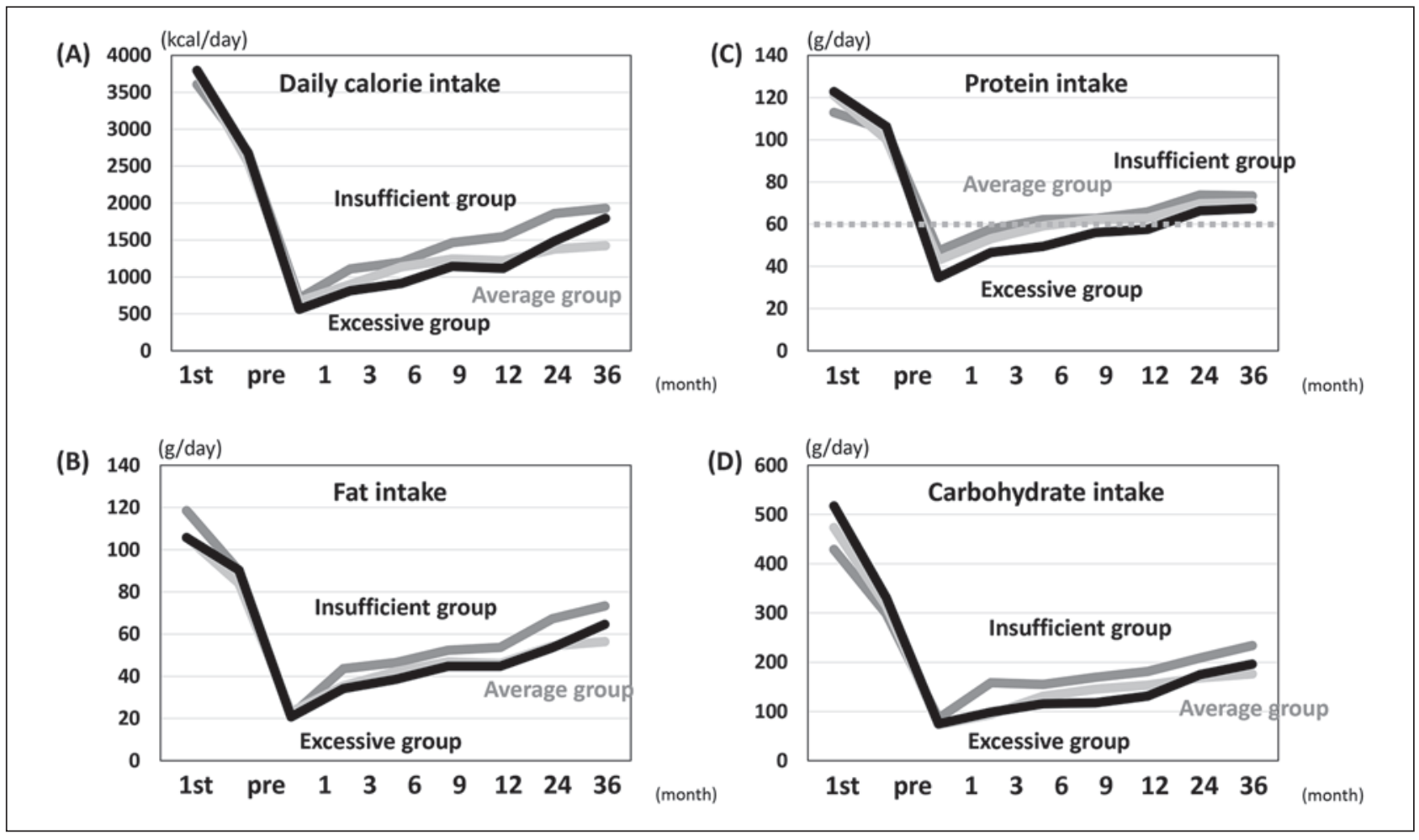

Fig. 5. Differences in postoperative nutrition intake among groups classified by 1 -year \%TWL. Changes in mean daily calorie (A), protein (B), fat (C) and carbohydrate (D) intakes are shown.

mental disorders and \%TWL in patients undergoing LSG [6]. The J-SMART study revealed that the prevalence of mental disorders was significantly higher both in the extremely low and high \%TWL groups. The present study examined the details of mental disorders, psychosocial aspects and nutritional status of patients after LSG classified by weight loss level. Our single-center study design, while being a weakness of this study, is also a strong point because we were able to study in detail the mental disorders and psychosocial aspects of all patients in this study.

The prevalence of mental disorders in this study was $51.7 \%$ and was higher than that reported by previous studies [7-9]. There were no significant differences in background parameters except for gender ratio, and in the changes in body weight, $\mathrm{HbA}_{1 \mathrm{c}}$ and daily calorie intake between the patients with and those without mental disorders. This study suggests 
Saiki et al.: Characteristics of Low and High \%TWL after LSG

that having mental disorders is not related to postoperative weight loss. Depression was reported to be the most common mental disorder in patients seeking and undergoing bariatric surgery [14]. A previous review concluded that one study found no difference in weight loss at 1 year but less weight loss at 4 years in patients with preoperative depression, but 4 other studies found no difference in postoperative weight loss between patients with and those without preoperative depression [14].

The U-shaped relationship between frequency of mental disorders and \%TWL was reported for the first time by J-SMART [6], and their finding is supported by the present study. A previous study reported that mood disorders were the most common mental disorder in obese individuals, followed by binge eating disorder and anxiety disorders [15]. In the present study, mood disorders were the most common mental disorder in all 3 groups classified by $\%$ TWL, followed by mental retardation/developmental disorders and anxiety disorders in the insufficient group, and by binge eating disorder and anxiety disorders in the excessive group. Furthermore, it is interesting that the BDI-II score tended to be higher in the insufficient and excessive groups (Table 3). Few studies have examined the relationship between intelligence and weight loss outcome. A previous study reported that patients with poorly controlled developmental disorders showed insufficient weight loss after bariatric surgery [16], and the prevalence of mental retardation/developmental disorders tended to be higher in patients with \%TWL $<15 \%$ in the J-SMART study [6]. Few studies have reported weight loss outcomes in patients with binge eating. A systematic review mentioned that binge eating is uncommon after bariatric surgery but often recurs when weight is regained [17]. This may be one of the reasons for weight regain after the initial excessive weight loss. Lower WAIS-III performance IQ is known to be associated with obesity [18], but the impact of performance IQ on weight loss outcome is unclear. Performance IQ reflects abilities related to visuospatial processes. In the present study, performance IQ tended to be lower in the insufficient and excessive groups than in the average group (Table 3). Lambda is an important variable in the Rorschach Comprehensive System, and persons with high Lambda are characterized as having an avoidant style of dealing with experience [19]. Lambda is known to be high in obese patients $[12,20]$. In this study, high lambda in the insufficient group may reflect the lack of a positive behavioral change.

This is also the first report that examines the effect of short-term \%TWL on mediumterm weight loss outcome. Interestingly, not only patients in the insufficient group, but also those in the excessive group showed weight regain after LSG. A similar tendency was observed for changes in VFA and $\mathrm{HbA}_{1 \mathrm{c}}$. A previous study observed that skeletal muscle volume decreases until 3 months after LSG and remains reduced until 12 months, with a decrease rate of around 10\% [21]. In the present study, SMM continued to decrease from 12 to 36 months after LSG in the insufficient group (-14.5\%) and the excessive group $(-15.0 \%)$. Focusing on the excessive group, there was a marked decrease in body weight in the short term followed by weight regain in the medium term and a decrease in SMM. After bariatric surgery, the recommended minimum protein intake is $60 \mathrm{~g} /$ day $[13,22]$. In the excessive group, it took 24 months for daily protein intake to reach $60 \mathrm{~g}$, which was longer than in the other groups. Therefore, SMM loss and weight regain may be due to protein deficiency. Formula diet contains high protein, low carbohydrate, low fat and sufficient vitamins and minerals, and is effective in reducing body weight [23] and maintaining or increasing skeletal muscle mass [24]. Formula diet is recommended before bariatric surgery as a very lowcalorie diet regime in order to improve perioperative outcomes [25]. Preoperative formula diet may also be effective to prevent protein deficiency and SMM loss after LSG. This possibility warrants further investigations. On the other hand, in the insufficient group, the frequency of snacking (so-called grazing), drinking habit and eating out may be related to insufficient weight loss. 
There are several limitations in this study. This study had a retrospective, observational design and was a single-center experience. Especially, the reasons for the high prevalence of mental disorders in this study may be not only that mental disorders were diagnosed preoperatively in all patients, but also that it was a single-center study. The sample size was small and could be a significant obstacle in identifying a trend and significant relationship. The statistical significance of the analyses of nutrition data was uncertain because of the difficulties to accurately calculate calorie and nutrient intake.

\section{Conclusion}

Although the prevalence of mental disorder in patients undergoing bariatric surgery has been known to be high, this study showed that the frequency of mental orders was particularly high in patients with insufficient weight loss (\%TWL $\leq 19.9 \%)$ and also in those with excessive weight loss ( $\%$ TWL $\geq 35.0 \%$ ). The problems observed in patients with insufficient weight loss were background mental health issues including mood disorders and intellectual disability, as well as postoperative eating behaviors including frequent snacking and eating out. In patients with excessive weight loss, high frequencies of depression and binge eating, as well as decrease in SMM due to low protein intake were observed. Furthermore, weight regain was observed 12 months after bariatric surgery both in patients with insufficient weight loss and in those with excessive weight loss. On the other hand, patients with average weight loss had fewer problems in weight loss outcome, mental health, nutrition intake and body composition. To improve long-term weight loss outcome and future health, a multidisciplinary approach focusing on mental health and nutrition is essential for patients undergoing bariatric surgery.

\section{Acknowledgment}

The authors wish to thank Aki Hagiwara for collection and assembly of data.

\section{Statement of Ethics}

All procedures and data collection were in accordance with the ethical standards of the institutional and Japanese national research committees or the ethical standards of the Helsinki Declaration of 1975. The protocol of the study was prepared in accordance with the Declaration of Helsinki and was approved by the Ethics Committee of Toho University Sakura Medical Center (S18061).

\section{Disclosure Statement}

All authors declare that they have no competing interests.

\section{Funding Sources}

There are no funding sources to declare.

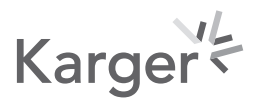




\section{Author Contributions}

R.K., S.N., S.T., R.O., Y.W., T.Y., M.O., T.O. and K.H. contributed to collection and/or assembly of data. A.S. contributed to the research concept and design, collection and/or assembly of data, data analysis and writing of the article. I.T. contributed to data interpretation and critical revision of the manuscript. All authors approved the version to be published.

\section{References}

1 Sacks FM, Bray GA, Carey VJ, Smith SR, Ryan DH, Anton SD, et al. Comparison of weight-loss diets with different compositions of fat, protein, and carbohydrates. N Engl J Med. 2009 Feb;360(9):859-73.

2 Pi-Sunyer X, Blackburn G, Brancati FL, Bray GA, Bright R, Clark JM, et al.; Look AHEAD Research Group. Reduction in weight and cardiovascular disease risk factors in individuals with type 2 diabetes: one-year results of the look AHEAD trial. Diabetes Care. 2007 Jun;30(6):1374-83.

3 Foley DL, Morley KI. Systematic review of early cardiometabolic outcomes of the first treated episode of psychosis. Arch Gen Psychiatry. 2011 Jun;68(6):609-16.

4 Tiihonen J, Lönnqvist J, Wahlbeck K, Klaukka T, Niskanen L, Tanskanen A, et al. 11-year follow-up of mortality in patients with schizophrenia: a population-based cohort study (FIN11 study). Lancet. 2009 Aug;374(9690): 620-7.

5 Angrisani L, Santonicola A, Iovino P, Vitiello A, Zundel N, Buchwald H, et al. Bariatric Surgery and Endoluminal Procedures: IFSO Worldwide Survey 2014. Obes Surg. 2017 Sep;27(9):2279-89.

6 Saiki A, Yamaguchi T, Tanaka S, Sasaki A, Naitoh T, Seto Y, et al.; Japanese Survey of Morbid and TreatmentResistant Obesity Group (J-SMART Group). Background characteristics and postoperative outcomes of insufficient weight loss after laparoscopic sleeve gastrectomy in Japanese patients. Ann Gastroenterol Surg. 2019 Aug;3(6):638-47.

7 Kalarchian MA, Marcus MD, Levine MD, Courcoulas AP, Pilkonis PA, Ringham RM, et al. Psychiatric disorders among bariatric surgery candidates: relationship to obesity and functional health status. Am J Psychiatry. 2007 Feb;164(2):328-34.

8 Mauri M, Rucci P, Calderone A, Santini F, Oppo A, Romano A, et al. Axis I and II disorders and quality of life in bariatric surgery candidates. J Clin Psychiatry. 2008 Feb;69(2):295-301.

9 Rosenberger PH, Henderson KE, Grilo CM. Psychiatric disorder comorbidity and association with eating disorders in bariatric surgery patients: A cross-sectional study using structured interview-based diagnosis. J Clin Psychiatry. 2006 Jul;67(7):1080-5.

10 Sullivan PA, Still CD, Jamieson ST, Dixon CB, Irving BA, Andreacci JL. Evaluation of multi-frequency bioelectrical impedance analysis for the assessment of body composition in individuals with obesity. Obes Sci Pract. 2018 Dec;5(2):141-7.

11 Mechanick JI, Kushner RF, Sugerman HJ, Gonzalez-Campoy JM, Collazo-Clavell ML, Spitz AF, et al.; American Society for Metabolic \& Bariatric Surgery. American Association of Clinical Endocrinologists, The Obesity Society, and American Society for Metabolic \& Bariatric Surgery medical guidelines for clinical practice for the perioperative nutritional, metabolic, and nonsurgical support of the bariatric surgery patient. Obesity (Silver Spring). 2009 Apr;17(Suppl 1):S1-70.

12 Mattlar CE, Salminen JK, Alanen E. Rorschach findings for the extremely obese: results from a two-year reducing programme. British Journal of Projective Psychology. 1989;34:2-27.

13 Mechanick JI, Youdim A, Jones DB, Timothy Garvey W, Hurley DL, Molly McMahon M, et al. Clinical practice guidelines for the perioperative nutritional, metabolic, and nonsurgical support of the bariatric surgery patient-2013 update: cosponsored by American Association of Clinical Endocrinologists, the Obesity Society, and American Society for Metabolic \& Bariatric Surgery. Surg Obes Relat Dis. 2013 Mar-Apr; $9(2)$ : 159-91.

14 Dawes AJ, Maggard-Gibbons M, Maher AR, Booth MJ, Miake-Lye I, Beroes JM, et al. Mental health conditions among patients seeking and undergoing bariatric surgery: a meta-analysis. JAMA. 2016 Jan;315(2):150-63.

15 Legenbauer T, De Zwaan M, Benecke A, Muhlhans B, Petrak F, Herpertz S. Depression and anxiety: their predictive function for weight loss in obese individuals. Obes Facts. 2009;2(4):227-34.

16 Williamson TM, Campbell TS, Telfer JA, Rash JA. Emotion self-regulation moderates the association between symptoms of ADHD and weight loss after bariatric surgery. Obes Surg. 2018 Jun;28(6):1553-61.

17 Spirou D, Raman J, Smith E. Psychological outcomes following surgical and endoscopic bariatric procedures: A systematic review. Obes Rev. 2020 Jun;21(6):e12998.

18 Yu ZB, Han SP, Cao XG, Guo XR. Intelligence in relation to obesity: a systematic review and meta-analysis. Obes Rev. 2010 Sep;11(9):656-70.

19 de Ruiter C, Cohen L. Personality in panic disorder with agoraphobia: a Rorschach study. J Pers Assess. 1992 Oct;59(2):304-16.

20 Elfhag K, Rossner S, Lindgren T, Andersson I, Carlsson AM. Rorschach personality predictors of weight loss with behavior modification in obesity treatment. J Pers Assess. 2004 Dec;83(3):293-305. 
21 Kenngott HG, Nickel F, Wise PA, Wagner F, Billeter AT, Nattenmüller J, et al. Weight loss and changes in adipose tissue and skeletal muscle volume after laparoscopic sleeve gastrectomy and Roux-en-Y gastric bypass: a prospective study with 12-month follow-up. Obes Surg. 2019 Dec;29(12):4018-28.

22 Steenackers N, Gesquiere I, Matthys C. The relevance of dietary protein after bariatric surgery: what do we know? Curr Opin Clin Nutr Metab Care. 2018 Jan;21(1):58-63.

23 Shirai K, Saiki A, Oikawa S, Teramoto T, Yamada N, Ishibashi S, et al. The effects of partial use of formula diet on weight reduction and metabolic variables in obese type 2 diabetic patients-multicenter trial. Obes Res Clin Pract. 2013 Jan-Feb;7(1):e43-54.

24 Watanabe Y, Kuribayashi N, Uchida D, Suzuki D, Kato M, Nagayama D, et al. Study protocol for the effects of formula diet with dapagliflozin on metabolic improvement and body composition in type 2 diabetes mellitus. Diabetes Ther. 2019 Feb;10(1):311-21.

25 Van Nieuwenhove Y, Dambrauskas Z, Campillo-Soto A, van Dielen F, Wiezer R, Janssen I, et al. Preoperative very low-calorie diet and operative outcome after laparoscopic gastric bypass: a randomized multicenter study. Arch Surg. 2011 Nov;146(11):1300-5. 